\title{
Reducing the Dosage: Decreasing Pediatric Clerkship Didactics
}

\author{
Amy Guiot*, Michael FitzGerald and Corinne Lehmann
}

Cincinnati Children's Hospital Medical Center, in affiliation with University of Cincinnati, College of Medicine in Cincinnati, Ohio

\begin{abstract}
Background: While case-based learning (CBL) sessions and Computer assisted Learning in Pediatrics Project (CLIPP) modules can be effective teaching modalities, there is little information about the optimal number and types of cases to require of medical students.

Aims: The aims of this investigation were to determine if a greater than 50\% reduction in CBL and CLIPP improved student perceptions regarding: 1) reasonableness of the time required to complete CBL and CLIPP 2) educational effectiveness of CBL and CLIPP 3) and assess potential impact on shelf exam scores.

Methods: The Class of 2013 completed 25 CBL and 6 CLIPP modules; a reduction from the 50 CBL and 16 CLIPP required of the 2012 class. A survey was emailed to students to assess their perceptions regarding the above aims.

Results: The class of 2013 indicated the number of CLIPP required was more reasonable. The two classes reported similar levels of perceived effectiveness. There was no difference in mean shelf exam scores.

Conclusion: We reduced by over 50\% the number of CBL and CLIPP without negatively impacting the perceived effectiveness of those methods or exam scores. Perhaps clerkship directors can be selective and more learner-centered when choosing required CBL or CLIPP.
\end{abstract}

Keywords: Computer Modules, Case Based Learning, Didactics, Pediatric Clerkship.

\section{INTRODUCTION}

Pediatric clerkships in the US and Canada commonly use case-based learning (CBL) discussion sessions and Computer-assisted Learning in Pediatrics Project (CLIPP) modules to provide consistent core curriculum knowledge [1]. The use of CBL sessions has been linked to increased satisfaction with the clerkship experience and improved scores on USMLE step 1 and 2 [2]. Students reported CLIPP modules to be effective learning tool [3].

Student comments from our clerkship evaluation indicated that although CBL cases and CLIPP modules were effective methods but redundant and time consuming at the same time. The search for information to help determine the optimal number and types of cases required for our trainees revealed little. Consequently, our education committee analyzed the Council of Medical Student Education in Pediatrics (COMSEP) curriculum objectives with our CBL cases, CLIPP modules, and logged student patient encounters to identify and remove cases that addressed commonly seen disease processes while maintaining core curricular content. We identified and eliminated over 50\% CBL and CLIPP found to be redundant. The aims of this investigation were to determine if a greater than $50 \%$

*Address correspondence to this author at the Cincinnati Children's Hospital Medical Center, 3244 Burnet Ave 5th floor ML 9016, Cincinnati, OH 45229; Tel: 513-636-4200; Cell: 513-253-6080;

E-mail: Amy.Guiot@cchmc.org reduction in CBL and CLIPP improved student perceptions regarding: 1) reasonableness of the time required to complete CBL and CLIPP; 2) educational effectiveness of CBL and CLIPP; 3) and potential impact on shelf exam scores.

\section{METHODS}

\section{Study Design}

This study used a non-equivalent control group post-test only with a custom-designed web-based survey created to measure perceptions regarding the reasonableness of the requirement and effectiveness of CBL and CLIPP. The control group consisted of students who completed $50 \mathrm{CBL}$ cases in 24-32 hours of small group sessions and 16 CLIPP. The intervention group consisted of students who completed $25 \mathrm{CBL}$ cases in 6 hours of sessions, and 6 CLIPP. The study was approved by the Cincinnati Children's Hospital Medical Center (CCHMC) Internal Review Board.

The reduction in the number of cases and time spent on case discussion between the class of 2012 and 2013 are detailed in Table $\mathbf{1}$.

\section{Study Participants}

Third year medical students from the University of Cincinnati College of Medicine (UCCOM) who participated in an eight-week pediatric clerkship at CCHMC were invited to participate. The control group included 79 students from the 2012 class on their pediatric clerkship January - June of 
Table 1. Reduction in CLIPP Modules and CBL Small Group Cases from Class of 2012 to Class of 2013.

\begin{tabular}{|l|c|c|}
\hline & Class of 2012 & Class of 2013 \\
\hline \hline Number of Required CLIPP & 16 & 6 \\
\hline Number of Required CBL small group cases & 50 & 25 \\
\hline Number of times per weekpulled off floor for discussions & $2-3$ times/week & 1 time/week \\
\hline Number of hours per week spend in discussion & $6-8$ hours/week & 4 hours/week \\
\hline
\end{tabular}

2011 and the intervention group included 83 students from the 2013 class on their pediatric clerkship January - June of 2012. Demographics between the classes were comparable in terms of males, females, minorities, undergraduate grade point average (GPA), Medical College Admission Test (MCAT) average, and United States Medical Licensing Exam (USMLE) Step 1 means. The demographics for the entire 2012 and 2013 classes are shown in Table 2.

\section{Survey Development and Administration}

The author-designed survey went through iterative drafts viewed by three educational experts within CCHMC, was pre-tested with a small group of fourth year medical students, and administered using Qualtrics ${ }^{\circledR}$ software, an online survey tool (https://www.qualtrics.com). An invitation and a survey link was emailed to students followed by periodic reminders over a four week period.The students received a $\$ 5.00$ coffee gift card in return for participating in the survey. By agreeing to answer the survey, the students consented to the project.
Demographics collected included year of graduation, month/year of pediatric clerkship, gender, and date of birth. Students rated the reasonableness of the amount of time spent preparing for CBL and CLIPP on a 7 point scale from 1 (very unreasonable) to 7 (very reasonable) and agreement regarding the effectiveness of CBL and CLIPP in providing new medical knowledge and improving clinical reasoning skills from 1 (completely disagree) to 7(completely agree). Students were also asked to rank seven activities when not attending a CBL session with one being the thing done most frequently and seven the least.

\section{Data Analyses}

Univariate descriptive statistics were reported for the Likert scale matrix items and yes/no questions as percentage frequency distributions. Bivariate t-tests were run using SPSS version 19 to analyze mean differences between academic years 2012 versus 2013 class on questions related to the reasonableness and effectiveness of CBL cases and CLIPP modules, on mean rankings of activities engaged in

Table 2. Demographic Comparison between the Classes of 2012 and 2013.

\begin{tabular}{|c|c|c|}
\hline & Class of 2012 & 166 \\
\hline \hline Total students per class & $159 \%$ & $89(54 \%)$ \\
\hline Males & $107(67 \%)$ & $77(46 \%)$ \\
\hline Females & $52(33 \%)$ & $10(6 \%)$ \\
\hline Minorities & $15(9 \%)$ & 3.58 \\
\hline Cumulative Undergraduate GPA & 3.6 & 32.4 \\
\hline MCAT Average & 32.3 & $79(48 \%)$ \\
\hline $23-25$ & & $62(37 \%)$ \\
$26-29$ & $71(45 \%)$ & $19(11 \%)$ \\
$30-35$ & $70(43 \%)$ & $5(3 \%)$ \\
$36-50$ & $12(8 \%)$ & $1(1 \%)$ \\
\hline USMLE Step 1 Mean & $4(3 \%)$ & 231 \\
\hline
\end{tabular}

Note. Since the study evaluated the students in the second half of the academic year January through June, the total number of students who were involved with the intervention was approximately half the total students per class. 
when not attending a CBL session, and on pediatric shelf exam scores.

\section{RESULTS}

Responses to the survey included 50/73 (68\%) of medical students from the class of 2012 and 53/82 (64\%) of medical students from the class of 2013. T-tests comparing the means between the class of 2012 and 2013 on the questions pertaining to the effectiveness of CBL cases and CLIPP modules revealed no statistically significant differences.

T-tests compared the means between the classes of 2012 and 2013 on responses regarding reasonableness of the time spent and reasonableness of the number of CBL and CLIPP. Means were based on a scale of 1 (very unreasonable) to 7 (very reasonable) and are reported in Table 3 . The one statistically significant difference item pertained to the reasonableness of the number of required CLIPP: the mean for the classes of 2013 of 5.42 was significantly higher than the mean of 4.77 for the class of 2012, $t(101)=-2.07, p$ $<.05$.

Regarding time spent on various activities when not attending CBL, the highest mean rank for both classes was "taking care of old patients" but the 2013 class mean of 1.78 was significantly higher than the 2012 class mean of 2.24, $t(102)=2.02, \mathrm{p}<.05$. See Table 4.

Respondents were also asked to rate the CBL cases and CLIPP modules in terms of helpfulness in preparing for the shelf exam on a scale from "Not at all helpful" $=1$ to "Extremely helpful" = 5. There was no significant difference between the two classes on their ratings for either CBL cases

Table 3. Mean Comparison between MD Graduate Years 2012 and 2013 Groups on Responses to Questions Regarding Reasonableness of the Time Spend and Number of Cases Required (Standard Deviations in Parentheses).

\begin{tabular}{|c|c|c|}
\hline & $2012(n=50)$ & $2013(n=53)$ \\
\hline The number of CLIPP modules I was required to complete. & $\begin{array}{c}4.77 \\
(1.72)\end{array}$ & $\begin{array}{c}5.42 \\
(1.59)\end{array}$ \\
\hline The number of CBL cases I was required to complete. & $\begin{array}{c}4.80 \\
(1.47)\end{array}$ & $\begin{array}{c}4.71 \\
(1.43)\end{array}$ \\
\hline The amount of time required to complete the CLIPP modules. & $\begin{array}{c}4.56 \\
(1.77)\end{array}$ & $\begin{array}{c}4.77 \\
(1.48)\end{array}$ \\
\hline The amount of time needed to fully prepare for the CBL case sessions. & $\begin{array}{c}4.82 \\
(1.41)\end{array}$ & $\begin{array}{c}4.49 \\
(1.49)\end{array}$ \\
\hline
\end{tabular}

Note. In bold $\mathrm{p}<.05$. Means are based on a scale of $1=$ Very unreasonable to $7=$ Very reasonable.

Table 4. Mean Ranking Comparison Between Classes of 2012 and 2013 on How Time is Spent When Not Attending CBL Small Group Conference.

\begin{tabular}{|l|c|c|}
\hline \multicolumn{1}{|c|}{ Activity } & Class of 2012 & Class of 2013 \\
\hline \hline Taking care of old patients & 2.24 & $1.78^{*}$ \\
\hline Admitting new patients & 2.78 & 2.30 \\
\hline Studying for the shelf exam & 2.70 & 2.81 \\
\hline Preparing for next small group case discussion & 3.50 & $4.16^{*}$ \\
\hline CLIPP cases & 4.73 & $5.26^{*}$ \\
\hline Personal time & 5.20 & 4.90 \\
\hline Other & 6.86 & 6.70 \\
\hline
\end{tabular}

Note. $* \mathrm{p}<.05 * * \mathrm{p}<.01$. Mean ratings are based on possible ratings of 1-7. 
or CLIPP modules. Mean ratings for the helpfulness of the CBL small group cases were 3.00 for the 2012 class and 3.06 for the 2013 class, $t(98)=-.30, \mathrm{p}=.763$. Mean ratings for the helpfulness of the CLIPP modules were 2.78 for the 2012 class and 2.96 for the 2013 class, $t(98)=-.75, \mathrm{p}=.450$.

Finally, the shelf exam means of 78.4 and 80.5 for 2012 and 2013 classes respectively were not significantly different, $\mathrm{t}(149)=-1.59, \mathrm{p}=0.113$.

\section{DISCUSSION}

We were able to reduce the number of CBL cases and CLIPP modules by over $50 \%$ without negatively impacting the perceived effectiveness of those methods or exam scores. One significant difference between the two classes was how the required number of CLIPP modules was perceived. CLIPP was created to fill gaps in curriculum using a national guideline in an effort to improve teaching and medical students education [4]. While the class of 2012 was assigned to a wide variety of 16 CLIPP modules, the class of 2013 was assigned with only six uncommonly seen pediatric topics. Fewer students in the class of 2013 perceived an unreasonable number to complete modules compared to the class of 2012. The ideal number of CLIPP modules is unknown to determine the optimal learning experience. Each program should assess the variety of patients students encounter during the clerkship as these same results may not apply to a smaller hospital which does not seen to be the same number of patients with such diverse disease entities or to a pediatric clerkship which is not eight weeks in duration.

Introducing CBL curriculum into the pediatric clinical clerkship was associated with improved shelf scores and student satisfaction [2]. Curtis' study used six cases of common pediatric illnesses not derived from the COMSEP curriculum; however, it is interesting to note that study used a handful of cases compared with our multitude. With an overwhelming majority from both classes the effectiveness of medical knowledge and clinical reasoning from small group CBL, it seems like a worthwhile time investment to have small group CBL but perhaps with a limited number. The ideal number of cases should be balanced, as CBL necessitates a time commitment for faculty and students to prepare and discuss. The extra obligation could mean less time taken for patient's care and preparing for the shelf exam. Perhaps a greater amount of time consuming to the clinical environment taking care of patients allows students to feel better prepared to deal with the challenges of patient care [5].

Our Class of 2013 ranked spending more time taking care of old patients compared with other responsibilities, for this class had at least 20 hours less CBL required session time and 10 less CLIPP assigned. The potential time difference in CBL and CLIPP preparation and completion could be over 40 hours per student. Fortunately, we did not detect a difference in mean shelf scores between the two classes, indicating that we were correct in our assessment of redundancies in our required CBL and CLIPP modules.
Perhaps clerkship directors can be more selective when choosing required CBL or CLIPP rather than assigning a multitude. Some institutions see a different variety of patients and may not need certain CBL or CLIPP to complement actual patient encounters. Hopefully, this curricular approach can be viewed as more learner-centered, allowing students more time to choose learning methods that best suit their learning style.

\section{LIMITATIONS}

The class of 2012 was sent a mass email requesting the survey by completed in January 2012. Students would have completed their pediatric clerkship anywhere from seven to twelve months prior to receiving the survey in comparison to the class of 2013 who received it on the day the clerkship would have completed. The qualitative data comments support memory as being an issue. Yet, the $68 \%$ and $64 \%$ participation of class correspondence is noteworthy. Perhaps the $\$ 5$ coffee gift card was a good incentive.

The original survey accidentally omitted the demographics which were not realized until after the first batch for the class of 2012 was emailed. We then had to send out an email requesting the students to re-submit their answers with the demographics included for which some students did not respond to the second survey requested. Our data from the class of 2012 who had to complete more small groups and CLIPP cases was, therefore, probably not according to our expectations.

\section{CONCLUSION}

This study demonstrated how we were able to reduce the number of CBL cases and CLIPP modules required of third year medical students on their pediatric clerkship by over $50 \%$ without negatively impacting the perceived effectiveness of those methods or exam scores. While this study was done at a large urban academic medical center, further studies would be needed to detect generalizability to medical students rotating at smaller institutions.

\section{CONFLICT OF INTEREST}

The authors confirm that this article content has no conflicts of interest.

\section{ACKNOWLEDGEMENTS}

Declared none.

\section{REFERENCES}

[1] Fall LH, Berman NB, Smith S, White CB, Woodhead JC, Olson AL. Computer-assisted learning in pediatrics project (clipp): multiinstitutional development and utilization of a computer-assisted learning program for the core pediatric clerkship. Acad Med 2005; 80(9): 847-55.

[2] Curtis JA, Indyk D, Taylor B. Successful use of problem-based learning in a third year pediatric clerkship. Ambul Pediatr 2001; 1: 132-35.

[3] Koestler J. 360 degrees: planning a new pediatric clerkship. Acad Med 2002; 77(11): 1163. 
[4] Schifferdecker KE, Berman NB, Fall LH, Fischer MR. Adoption of computer-assisted learning in medical education: The educator's perspective. Med Edu 2012; 46(11), 1063-73.
[5] Yu F, Xu L, Lu D, Luo W, Wang Q. The Integrated Clerkship: An innovative model for delivering clinical education at the zhejiang university school of medicine. Acad Med 2009; 84(7): 886-94.

Received: February 20, 2014

Revised: March 04, 2014

Accepted: March 07, 2013

(C) Guiot et al.; Licensee Bentham Open.

This is an open access article licensed under the terms of the Creative Commons Attribution Non-Commercial License (http://creativecommons.org/licenses/by-nc/3.0/) which permits unrestricted, non-commercial use, distribution and reproduction in any medium, provided the work is properly cited. 